Freiburg-THEP 96/6

April 1996

\title{
On the evaluation of three-loop scalar integrals in the massive case
}

\author{
Adrian Ghinculov \\ Albert-Ludwigs-Universität Freiburg, Fakultät für Physik \\ Hermann-Herder Str.3, D-79104 Freiburg, Germany
}

\begin{abstract}
I describe a method to calculate a class of three-loop selfenergy diagrams for arbitrary internal masses and external momentum. This method combines analytical results and numerical integration, and is suitable for implementation in a computer program to produce fast and accurate results. For the class of Feynman diagrams considered in this paper this method leads to a two-fold integral which needs to be evaluated numerically. Results are given for a range of masses and external momentum.
\end{abstract}




\section{Introduction}

Perturbation theory based on Feynman diagrams continues to be the main tool for calculating observable quantities which can be measured in high energy experiments. Unfortunately, the difficulty of the calculations increases dramatically with the loop order.

Some electroweak parameters are being measured in LEP/SLC experiments at the per mille level. To match such accuracy, full two-loop electroweak corrections may be needed in the future [1]. Higher loop contributions are also needed when one deals with relatively large couplings. Here higher order corrections can be used to increase the accuracy of the calculation and to estimate the validity range of perturbation theory. In the electroweak sector potentially large radiative corrections may be induced for instance by the large Yukawa coupling of the top quark, and also if the mass of the minimal Higgs boson turns out to be large. One can also imagine extensions of the symmetry breaking sector of the standard model where strong interactions may play an essential rôle [2].

In calculating radiative corrections, the difficult step is usually the evaluation of the scalar integrals.

At one-loop order this is in principle a completely solved problem. However, in practice the evaluation of one-loop scalar integrals in the general mass case can be tricky because one has to make sure the functions involved remain on the physical Riemann sheet, and because of the need to control potentially large numerical cancellations [3].

At two-loop level, well-known results by van der Bij and Veltman guarantee that all vacuum diagrams with arbitrary masses can be expressed analytically in terms of Spence functions 沕. If the external momenta do not vanish, this is in general not possible any longer. In fact, the twoloop selfenergy diagram with three propagators is known to be related to the Lauricella function, which is a generalization of the hypergeometric series [5]. Such functions are not straightforward to evaluate. Typically they are expressed by multiple series whose convergence may be poor for certain choices of the parameters, for instance near a threshold. Therefore one has to rely at the two-loop level at least partly on numerical techniques. A combination of analytical and numerical methods exists which can be used in principle to calculate any two-loop diagram. There exist also a large number of techniques which work for certain topologies or for special choices of parameters. On the evaluation of two-loop scalar integrals in the general massive case see refs. [5]-[14]. 
While techniques to handle massless three-loop diagrams exist [15], the massive case is much more difficult. Apart from a formal expression for the four propagator selfenergy diagram [5], no general results exist at the three-loop level. Considering the previous remarks on the massive two-loop diagrams, there is little hope that analytical techniques can prove useful for realistic three-loop calculations of physical relevance in the massive case.

It is the purpose of this letter to provide efficient methods for evaluating certain massive three-loop diagrams. Instead of trying to solve the general problem, this paper limits itself to calculating the selfenergy diagram of fig. 1. With little modifications, this method can be used to calculate some other diagrams, of the type shown in fig. 2 .

The scalar integral corresponding to the diagram of fig. 1 is:

$$
\begin{aligned}
& I\left(m_{1}^{2}, m_{2}^{2}, \ldots, m_{8}^{2} ; k^{2}\right)= \\
& \quad \int d^{n} p d^{n} q d^{n} r \frac{1}{\left(q^{2}-m_{1}^{2}\right)\left[(q+k)^{2}-m_{2}^{2}\right]} \frac{1}{\left(r^{2}-m_{4}^{2}\right)\left[(r-k)^{2}-m_{5}^{2}\right]} \\
& \quad \frac{1}{\left(p^{2}-m_{7}^{2}\right)\left[(p+k)^{2}-m_{8}^{2}\right]} \frac{1}{\left[(p-q)^{2}-m_{3}^{2}\right]\left[(p+r)^{2}-m_{6}^{2}\right]} \cdot
\end{aligned}
$$

To calculate this diagram one starts by performing the integrals over $q$ and $r$ analytically, and leaves the $p$ integral to be performed numerically after an examination of the analytical structure of the integrand.

The $q$ and $r$ integrals are essentially two one-loop triangle subdiagrams. Of course, in our case two of the external momenta of the triangle subdiagrams are functions of the loop momentum $p$ of the three-loop diagram. To fix the notations, let us denote:

$$
\begin{aligned}
& C\left(m_{1}^{2}, m_{2}^{2}, m_{3}^{2} ; p_{1}^{2}, p_{2}^{2}, p_{3}^{2}\right)= \\
& \quad \int d^{n} p \frac{1}{\left(p^{2}-m_{1}^{2}\right)\left[\left(p-p_{2}\right)^{2}-m_{3}^{2}\right]\left[\left(p+p_{3}\right)^{2}-m_{2}^{2}\right]} .
\end{aligned}
$$

Such triangle graphs are expressible in terms of Spence functions for any choice of the internal masses and external momenta. Explicit formulae can be found for instance in refs. [17] or [18]. These expressions were encoded in the program FF [19]. This program will be used in the following to calculate the diagram of fig. 1 in the general mass case. However, the rather lengthy expression of the function $C$ simplifies considerably for special choices of the 
masses. Such is for instance the massles case, $m_{1}=m_{2}=m_{3}=0$. In this case a formula can be derived which is better suited for numerical evaluation than the general case formula used in FF. Equivalent formulae were obtained previously by other authors (see for instance ref. [16]), but the expression which will be derived in the following has the advantage of remaining on the right sheet for the parameter range needed in this three-loop calculation. We therefore sketch the derivation of this formula.

One introduces Feynman parameters, performs the integral over the loop momentum, expands in $\epsilon=n-4$, integrates over one Feynman parameter, and obtains:

$$
C\left(0,0,0 ; p_{1}^{2}, p_{2}^{2}, p_{3}^{2}\right)=-i \frac{\pi^{2}}{p_{3}^{2}} \int_{0}^{1} d x\left[\frac{1}{x(1-x)\left(1-\mu^{2}\right)} \log \mu^{2}\right],
$$

where

$$
\begin{aligned}
\mu^{2} & =\frac{a x+b(1-x)}{x(1-x)} \\
a & =\frac{p_{1}^{2}}{p_{3}^{2}}, \quad b=\frac{p_{2}^{2}}{p_{3}^{2}}, \quad .
\end{aligned}
$$

It suffices to keep only the $\mathcal{O}\left(\epsilon^{0}\right)$ terms in the expression of $C$ because the three-loop diagram of eq. 1 is obviously ultraviolet convergent. The integral of eq. 3 can be carried out analytically and gives:

$$
\begin{aligned}
& C\left(0,0,0 ; p_{1}^{2}, p_{2}^{2}, p_{3}^{2}\right)= \\
& \begin{array}{c}
\frac{2 i \pi^{2}}{p_{3}^{2} \sqrt{\Delta}}\left[S p\left(-\frac{u_{2}}{v_{1}}\right)+S p\left(-\frac{v_{2}}{u_{1}}\right)+\frac{1}{4} \log ^{2} \frac{u_{2}}{v_{1}}+\frac{1}{4} \log ^{2} \frac{v_{2}}{u_{1}}\right. \\
\left.+\frac{1}{4} \log ^{2} \frac{u_{1}}{v_{1}}-\frac{1}{4} \log ^{2} \frac{u_{2}}{v_{2}}+\frac{\pi^{2}}{6}\right],
\end{array}
\end{aligned}
$$

where

$$
\begin{aligned}
u_{1,2} & =\frac{1}{2}(1+b-a \pm \sqrt{\Delta}) \\
v_{1,2} & =\frac{1}{2}(1-b+a \pm \sqrt{\Delta}) \\
\Delta & =1-2(a+b)+(a-b)^{2} .
\end{aligned}
$$


This function is similar to the finite part of the two-loop integral $\mathcal{G}\left(m_{1}, m_{2}, m_{3} ; 0\right)$ of ref. [6], or $\left(m_{1}, m_{1}\left|m_{2}\right| m_{3}\right)$ of ref. [4]. This is not surprising, since there is a relation between one-loop massless diagrams evaluated at finite external momentum and massive two-loop vacuum diagrams. Other relations of this type were obtained for instance in ref. [16].

One can now write the three-loop diagram of fig. 1 in the following form:

$$
\begin{aligned}
& I\left(m_{1}^{2}, m_{2}^{2}, \ldots, m_{8}^{2} ; k^{2}\right)= \\
& \int d^{4} p \frac{1}{\left(P_{1}^{2}-m_{7}^{2}\right)\left[P_{2}^{2}-m_{8}^{2}\right]} C\left(m_{1}^{2}, m_{2}^{2}, m_{3}^{2} ; P_{2}^{2}, P_{1}^{2}, k^{2}\right) \\
& \times C\left(m_{4}^{2}, m_{5}^{2}, m_{6}^{2} ; P_{2}^{2}, P_{1}^{2}, k^{2}\right),
\end{aligned}
$$

where

$$
\begin{aligned}
& P_{1}^{2}=p^{2} \\
& P_{2}^{2}=(p+k)^{2} .
\end{aligned}
$$

This expression has the structure of a one-loop integral with some vertex form factors. As already mentioned, one does not need to keep terms of order $\epsilon$ and higher in the expression of $C$ because $I$ is ultraviolet finite. One can choose the external momentum to have vanishing space components, $k=\left(\sqrt{k^{2}}, \overrightarrow{0}\right)$. Let us denote the time and space components of the loop momentum by $p \equiv\left(p_{0}, \vec{p}\right)$. It is obvious that the integrand in eq. 7 is independent of the direction of $\vec{p}$. Therefore one can readily perform the angular integration over the direction of $\vec{p}$. The result reads:

$$
\begin{aligned}
& I\left(m_{1}^{2}, m_{2}^{2}, \ldots, m_{8}^{2} ; k^{2}\right)= \\
& \qquad \int_{-\infty}^{\infty} d p_{0} \int_{0}^{\infty} d \rho \frac{4 \pi \rho^{2}}{\left(P_{1}^{2}-m_{7}^{2}\right)\left[P_{2}^{2}-m_{8}^{2}\right]} C\left(m_{1}^{2}, m_{2}^{2}, m_{3}^{2} ; P_{2}^{2}, P_{1}^{2}, k^{2}\right) \\
& \quad \times C\left(m_{4}^{2}, m_{5}^{2}, m_{6}^{2} ; P_{2}^{2}, P_{1}^{2}, k^{2}\right)
\end{aligned}
$$

where

$$
\begin{aligned}
& P_{1}^{2}=p_{0}^{2}-\rho^{2} \\
& P_{2}^{2}=p_{0}^{2}+2 p_{0} \sqrt{k^{2}}+k^{2}-\rho^{2}
\end{aligned}
$$


and $\rho=|\vec{p}|$.

In this way one has obtained a two-fold integral representation of the three-loop diagram shown in fig. 1.

Some remarks on the numerical integration are now in place.

The possible singularities of the integrand in the integration domain $\left(p_{0}, \rho\right) \in(-\infty,+\infty) \times(0,+\infty)$ are given by the solutions of the equations:

$$
\begin{aligned}
p_{0}^{2}-\rho^{2}-m_{7}^{2}+i \eta & =0 \\
p_{0}^{2}+2 p_{0} k+k^{2}-\rho^{2}-m_{8}^{2}+i \eta & =0
\end{aligned}
$$

and by the thresholds of the vertex functions $C$. The threshold behaviour of the functions $\mathrm{C}$ does not pose special problems with respect to the numerical integration of expression 9 because the integrand remains finite at these points. One also has to keep in mind that for special values of the mass and momentum parameters, additional problems may come from the infrared singularities of the function $C . C\left(m_{1}^{2}, m_{2}^{2}, m_{3}^{2} ; p_{1}^{2}, p_{2}^{2}, p_{3}^{2}\right)$ displays an infrared singularity for $p_{3}^{2}=m_{2}^{2}, p_{2}^{2}=m_{3}^{2}$ and $m_{1}^{2}=0$ and cyclic permutations.

The simplest and most efficient way to avoid the singularities associated with eqns. 11 is to perform a Wick rotation of the loop momentum $p$. Instead of the usual rotation of the time component $p_{0}$, it is more convenient to rotate the radial space component $\rho$ with a negative phase $e^{-i \alpha}$, with $\alpha \in(0, \pi / 2)$. This way the integrand becomes free of singularities and can be integrated easily. At the same time this procedure allows a useful check on the calculation because the result must be independent of the actual value of $\alpha$.

One can also calculate this integral without introducing a complex $\rho$. To do this, the integral has to be split along the solutions of eqns. 11. Without this factorization of the singularities, the adaptative integration algorithms are rather inefficient because the singularities are smeared across both integration variables $p_{0}$ and $\rho$.

As a check on the calculation, one notices that modifying formula 9 into:

$$
\begin{aligned}
& J\left(m_{1}^{2}, m_{2}^{2}, \ldots, m_{5}^{2} ; k^{2}\right)= \\
& \left.\quad \int_{-\infty}^{\infty} d p_{0} \int_{0}^{\infty} d \rho \frac{4 \pi \rho^{2}}{\left(P_{1}^{2}-m_{4}^{2}\right)\left[P_{2}^{2}-m_{5}^{2}\right]} C\left(m_{1}^{2}, m_{2}^{2}, m_{3}^{2} ; P_{2}^{2}, P_{1}^{2}, k^{2}\right) 12\right)
\end{aligned}
$$

gives the two-loop selfenergy diagram shown in fig. 3 , which was calculated 
by a number of authors. For $m_{1}=m_{2}=m_{3}=0, m_{4}=m_{5}=|k|=1$ the result is:

$$
\begin{aligned}
& J(0,0,0,1,1 ; 1)= \\
& \quad \pi^{4}\left[\left(1.8472631 \pm 1.8 \cdot 10^{-6}\right)+i\left(3.4451413 \pm 1.8 \cdot 10^{-6}\right)\right],
\end{aligned}
$$

in agreement with already existing results [6, 7]. The case $m_{1}=m_{2}=m_{3}=$ $m_{4}=m_{5}=|k|=1$ gives:

$$
J(1,1,1,1,1 ; 1)=\pi^{4}\left[\left(.923663 \pm 2.6 \cdot 10^{-5}\right)\right],
$$

which again agrees with the known value of this diagram [6, [7].

Turning now to the three-loop case, fig. 4 shows the results for the selfenergy diagram of fig. 1 for $m_{1}=m_{2}=m_{3}=m_{4}=m_{5}=m_{6}=0$, $m_{7}=m_{8}=1$ as a function of the external momentum $k^{2}$. It was checked that both eq. 5 and the general formula encoded in FF lead to the same results. Fig. 5 shows the same diagram in the all-massive case $m_{1}=m_{2}=$ $m_{3}=m_{4}=m_{5}=m_{6}=1 / 2, m_{7}=m_{8}=1$.

Obviously, it is straightforward to modify the formula 9 for calculating other three-loop diagrams of the type shown in fig. 2. In a similar way, it is also possible to introduce more propagators in eq. 12 for calculating a few other two-loop scalar integrals. For example, fig. 6 shows the results for diagram 2 a) in the case $m_{1}=m_{2}=m_{3}=m_{4}=m_{5}=m_{6}=0, m_{7}=1$.

To conclude, a simple method was described which allows one to calculate efficiently a class of three-loop selfenergy diagrams. It works for any masses and momentum combinations in the physical region. As a byproduct, this yields a new method for calculating certain two-loop Feynman diagrams.

\section{Acknowledgement}

I am grateful to the theory department of Brookhaven National Laboratory, where this paper was written during a short visit, for hospitality. This research was supported by the Deutsche Forschungsgemeinschaft (DFG).

\section{References}

[1] Reports of the Working Group on Precision Calculations for the Z Resonance, D. Bardin, W. Hollik, G. Passarino editors, CERN 95-03 (1995). 
[2] A. Hill and J.J. van der Bij, Phys. Rev. D36 (1987) 3463.

[3] G.J. van Oldenborgh and J.A.M. Vermaseren, Z. Phys C46 (1990) 425.

[4] J.J. van der Bij and M. Veltman, Nucl. Phys. B231 (1984) 205.

[5] F.A. Berends, M. Buza, M. Böhm and R. Scharf, Z. Phys. C63 (1994) $22 \%$.

[6] A. Ghinculov and J.J. van der Bij, Nucl. Phys. B436 (1995) 30; A. Ghinculov, Phys. Lett. B337 (1994) 137; (E) B346 (1995) 426; Nucl. Phys. B455 (1995) 21 and references therein.

[7] L. Durand, B.A. Kniehl and K. Riesselmann, Phys. Rev. D51 (1995) 5007; Phys. Rev. Lett. 72 (1994) 2534; (E) Phys. Rev. Lett. 74 (1995) 1699.

[8] R. Scharf, J.B. Tausk, Nucl. Phys. B412 (1994) 523.

[9] D. Kreimer, Phys. Lett. B273 (1991) 27r; Phys. Lett. B292 (1992) 341. A. Czarnecki, U. Kilian, D. Kreimer, Nucl. Phys. B433 (1995) 259.

[10] F.A. Berends and J.B. Tausk, Nucl. Phys. B421 (1994) 456; F.A. Berends, A.I. Davydychev, V.A. Smirnov, J.B. Tausk, Nucl. Phys. B439 (1995) 536.

[11] S. Bauberger, F.A. Berends, M. Bohm, M. Buza, Nucl. Phys. B434 (1995) 383.

[12] A.I. Davydychev and J.B. Tausk, Nucl. Phys. B397 (1993) 123; A.I. Davydychev, V.A. Smirnov and J.B. Tausk, Nucl. Phys. B410 (1993) 325.

[13] J. Fleischer and O.V. Tarasov, Z. Phys. C64 (1994) 413.

[14] J. Fujimoto, Y. Shimizu, K. Kato, Y. Oyanagi, KEK-92-213 (1992); J. Fujimoto, Y. Shimizu, K. Kato, T. Kaneko, KEK-CP-028 (1995).

[15] S.A. Larin, F.V. Tkachev, J.A.M. Vermaseren, NIKHEF-H/91-18 (1991).

[16] N.I. Usyukina, A.I. Davydychev, Phys. Lett. B332 (1994) 159. 
[17] G. 't Hooft and M. Veltman, Nucl. Phys. B153 (1979) 365.

[18] A. Denner, Fortschr. Phys. 41 (1993) $30 \%$.

[19] G.J. van Oldenborgh, Comput. Phys. Commun. 66 (1991) 1. 


\section{Figure captions}

Fig.1 Three-loop selfenergy diagram in the general massive case.

Fig.2 Some three-loop diagrams which can be calculated with the same methods as the diagram in fig. 1 . The dots on internal lines are external vertices connected to zero external momenta.

Fig.3 A two-loop selfenergy diagram which can be calculated with the same methods as the three-loop diagram of fig. 1 .

Fig. 4 The real (solid line) and the imaginary (dashed line) parts of the scalar three-loop function $\pi^{6} I$, with $I$ defined in eq. 1 , as a function of the external momentum squared $k^{2}$. The masses of the internal lines are $m_{1}=m_{2}=m_{3}=m_{4}=m_{5}=m_{6}=0, m_{7}=m_{8}=1$. Note that due to the definition of $I$ the absorbtive part of the corresponding diagram is the real part of $I$.

Fig. 5 Same as fig. 4, but for the all-massive case $m_{1}=m_{2}=m_{3}=$ $m_{4}=m_{5}=m_{6}=1 / 2, m_{7}=m_{8}=1$.

Fig. 6 Same as fig. 4, but for the three-loop diagram of fig. 2 a) in the case case $m_{1}=m_{2}=m_{3}=m_{4}=m_{5}=m_{6}=0, m_{7}=1$. 